\title{
Calculating risk changes after negative mutation test outcomes for autosomal dominant hereditary late-onset disorders
}

\author{
B Bonke ${ }^{1}$, A Tibben ${ }^{2}$, D Lindhout ${ }^{3}$ and T Stijnen ${ }^{4}$ \\ ${ }^{1}$ Department of Medical Psychology \& Psychotherapy, Netherlands Institute of Health Sciences, Erasmus MC, PO Box 1738, Rotterdam \\ 3000 DR, The Netherlands; ${ }^{2}$ Departments of Clinical Genetics and Neurology, Leiden University Medical Centre, Leiden, The \\ Netherlands; ${ }^{3}$ Department of Medical Genetics, University Medical Centre Utrecht, Utrecht, The Netherlands; ${ }^{4}$ Department of \\ Epidemiology and Biostatistics, Erasmus MC, Rotterdam, The Netherlands
}

\begin{abstract}
We demonstrate, in a specific scenario, the effect of negative test results from relatives in families at risk for an autosomal dominant hereditary late-onset disorder. A hypothetical pedigree, of a family at risk of Huntington's disease, was used to demonstrate the consequences for the risk status of various family members in the case where relatives have been tested, and found to be mutation negative. We argue that accurate
\end{abstract}

assessment of conditional probabilities in clinical genetics is important for individuals at risk for hereditary disorders with Mendelian transmission patterns; our formulae offer the opportunity - when simplifying assumptions are met - to determine the changed risk status of individuals in such cases. Heredity (2006) 96, 259-261. doi:10.1038/sj.hdy.6800790; published online 4 January 2006

Keywords: hereditary late-onset disorders; Huntington's disease; negative test outcome; predictive testing; Bayesian statistics

\section{Introduction}

Conditional probabilities in genetic risk estimation are notoriously difficult to grasp. This was recently demonstrated in a survey among healthcare professionals who estimated risks in cases where certain relatives were established to be noncarriers of an autosomal dominant hereditary late-onset disease such as Huntington's disease (HD) (Bonke et al, 2002, 2005). Risk estimate is even more difficult in cases with complicated pedigrees where more remote relatives are noncarriers. As an aid to clinical geneticists and other healthcare professionals in clinical genetics, we have drawn up a hypothetical pedigree to illustrate how such risk estimations can be made in these more complicated situations (Figure 1). Our objective is to demonstrate the magnitude of the effect of negative test results on relatives. In this simplified scenario, one individual in generation I was affected by HD.

\section{Methods and results}

Depending on the position in the pedigree, family members at risk have different prior risks, varying from $50 \%$ in generation II to $12.5 \%$ in generation IV. Suppose that an individual at $25 \%$ risk (eg, individual III:4 in Figure 1) - in a pedigree similar to the one presented with a parent at $50 \%$ risk without signs of the disease

Correspondence: B Bonke, Department of Medical Psychology $\mathcal{E}$ Psychotherapy, Netherlands Institute of Health Sciences, Erasmus MC, PO Box 1738, Rotterdam 3000 DR, The Netherlands;

E-mail: b.bonke@erasmusmc.nl

Received 12 May 2005; accepted 14 November 2005; published online 4 January 2006 considers having children, but neither wishes to know his or her personal risk nor wishes to pass the gene on to the child. Consequently, the parent chooses prenatal mutation testing, accepting the chance that the test is positive. A positive test result for the unborn would lead to a termination of pregnancy and would determine the carrier status of the parent and the grandparent. A negative test result, however, provides otherwise important information for many relatives of the foetus: it diminishes their risks provided that no other test information - and particularly no positive test results is available and clinical signs of the disease are absent. The more the individuals in this generation who have tested negative, the more the probabilities of being a carrier diminish for their relatives. For instance, the prior risk for individuals IV: 1 and IV:5 is $12.5 \%$; if prenatal tests for individuals IV:2, IV:3, and IV:4 have all been negative, with no other test results available, the risks for individuals IV:1 and IV:5 of being a carrier will have diminished to 9 and 2\%, respectively. Such negative test outcomes are also relevant for uncles or aunts of these tested individuals: the risk of individual III:5, for instance, decreases from 25 to $18 \%$. This is particularly relevant if they consider raising a family. We present formulae, derived from Bayes' theorem (Bridge, 1994; Gelehrter et al, 1998), for calculating risks if prenatal testing has shown that $n$ descendants of individuals II:1 and III: 4 in the youngest generation are noncarriers (Figure 1 and Table 1). These formulae do not take age effects into account, but evidently the probability of being a carrier diminishes as time goes by without clinical symptoms (Claus et al, 1993; Maat-Kievit et al, 2002). If the age-specific penetrance of a disorder is 
known, then the formulae must be adjusted for age effects, as described below. More complicated situations occur for an individual originally at 25\% risk (eg, individual III:2) with one or more siblings, nieces, or nephews, all tested negative. The risk of individual III:2 then diminishes with each new negative test outcome. Likewise, the risk of a descendant of individuals II:1 and III:4 in the youngest generation (ie, the unborn IV:5) with one or more siblings, uncles, or aunts, all tested negative, is substantially lower than $12.5 \%$. In cases where only one or more of the individuals III:2, III:4, III:5 at 25\% risk have tested negative, the risk for individual II:1 diminishes (ie, probability of individual II:1 being a gene carrier equals $1 /\left(2^{n}+1\right)$ for $n$ of her children tested negative). The changed risks for relatives in generations

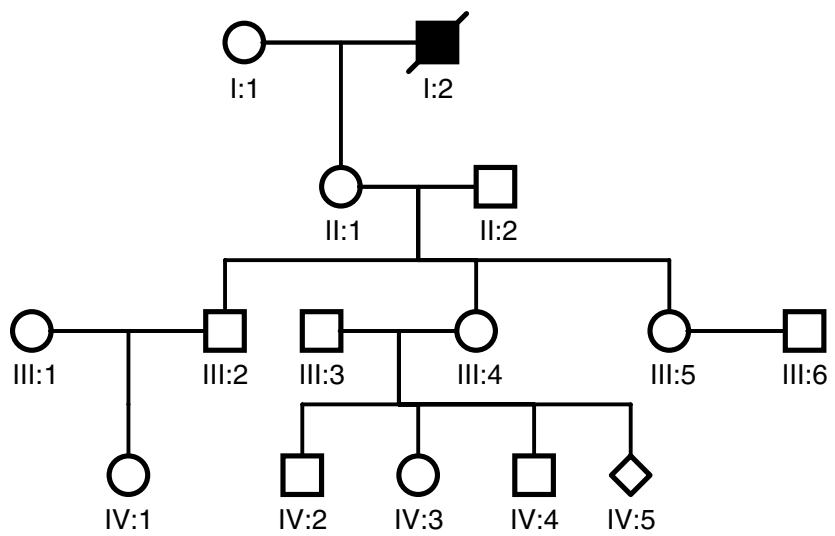

Figure 1 Hypothetical pedigree with three generations at risk for an autosomal dominant hereditary late-onset disease (in this case: HD) The older generation contains an affected individual (I:2), the next generation one individual at $50 \%$ risk (II:1). For the sake of convenience, it is assumed that individual II:1 is either relatively young and without clinical signs of the disease, or has died without showing such signs. The next generation contains three individuals at $25 \%$ risk (III:2, III:4, III:5), and the youngest generation five at $12.5 \%$ risk (IV:1-IV:5). If prenatal tests for one or more of the descendants of individuals II: 1 and III:4 in the youngest generation (IV:2, IV:3, IV:4, respectively) have been negative, the calculated carrier risks of their parent (III:4) and grandparent (II:1) at risk, of any untested (future) siblings (IV:5), and of aunts, uncles, and cousins at risk (III:2, III:5, IV:1) decrease as compared with the risks before testing. All blank symbols represent individuals who are lacking clinical signs of the disease.
III and IV can then be obtained easily by multiplication (by 0.5 and 0.25 , respectively). The formulae in Table 1 are presented as points of departure for calculating the changed risks, under simplifying assumptions, when all other factors that determine risks are known. It is important to note that the functions (formulae) have different limits with increasing $n$ (see Table 1). Those for first-degree relatives approach the value 0 for $n \rightarrow \infty$, which one would expect from the pedigree. The functions for other relatives have a limit for $n \rightarrow \infty$ that corresponds to the situation where individual III:4 is an established noncarrier. In that case, $\mathrm{P}(\mathrm{II}: 1=$ carrier $)=$ $33.3 \%$ and hence P(III: 2 or III: $5=$ carrier $)=16.7 \%$ and $\mathrm{P}(\mathrm{IV}: 1=$ carrier $)=8.33 \%$ (Bonke et al, 2002).

\section{Discussion}

With increasing age and absence of clinical signs or symptoms, the probability of individual II:1 being a carrier of the HD gene diminishes. Instead of the theoretical (prior) probability of 0.5 , this changed probability will be anywhere between 0 and 0.5 . What happens to our formulae if one takes age-dependent penetrance into account by, for instance, estimating the age-adjusted risk through the use of life table risks (Harper and Newcombe, 1992)? Let $p$ be the estimated age-adjusted probability that individual II:1 is a carrier of the HD gene. Then the probability that individual II:1 is a carrier given that $n$ children of individual III:4 in Figure 1 are established noncarriers and no children were tested positive - can be calculated by using Bayes' theorem as $p *\left(1+2^{n}\right) /\left[p *\left(1-2^{n}\right)+2^{n+1}\right]$ (Table 2). It is possible, in principle, to adjust for age-dependent penetrance for all relevant individuals in the pedigree in an analogous way, but the formulae then become exceedingly complicated. All scenarios presented here can, however, be solved and general instructions for calculating probabilities for these and other situations are available upon request. Of course, all risks change dramatically as soon as a relative has received a positive test outcome or shows symptoms of the disease. It is important to note that our formulae and derivations are based on the assumption that there is a $100 \%$ detection rate for the mutation being tested, as is more or less true for HD. In cases where the sensitivity and/or specificity are below $100 \%$, this must be accounted for in the calculations.

Table 1 Probabilities, in percentages, of being an HD carrier for various relatives in Figure 1, if prenatal mutation tests for $n$ children of individual III:4 have been negative, with relevant formulae for calculating changed risks

\begin{tabular}{llcccccc}
\hline & Individuals in pedigree & $\mathrm{n}=0$ (prior) & $\mathrm{n}=1$ & $\mathrm{n}=2$ & $\mathrm{n}=3$ & Formula $\mathrm{P}(\mathrm{C})$ & Limit for $\mathrm{n} \rightarrow \infty$ \\
\hline First-degree relatives & III:4 & 25 & $14.3^{\mathrm{a}}$ & 7.7 & 4 & $1 /\left(3 * 2^{n}+1\right)$ & 0 \\
& IV:5 & 12.5 & 7.1 & 3.8 & 2 & $1 /\left(6 * 2^{n}+2\right)$ & 0 \\
Second- and third-degree relatives & II:1 & & & & & \\
& III:2, III:5 & 50 & 42.9 & 38.5 & 36 & $\left(2^{n}+1\right) /\left(3 * 2^{n}+1\right)$ & 33.3 \\
& IV:1 & 25 & 21.4 & 19.2 & 18 & $\left(2^{n}+1\right) /\left(6 * 2^{n}+2\right)$ & 16.7 \\
& 12.5 & 10.7 & 9.6 & 9 & $\left(2^{n}+1\right) /\left(12 * 2^{n}+4\right)$ & 8.33 \\
\hline
\end{tabular}

$n=$ number of descendants of individuals II:1 and III:4 in the youngest generation (ie, individuals IV:2, IV:3, and IV:4, respectively) for whom prenatal mutation tests have been negative; $P(\mathrm{C})=$ probability of being a carrier.

${ }^{\mathrm{a}}$ For example, using the relevant formula for $n=1$ : $\mathrm{P}(\mathrm{C})=1 /\left(3 * 2^{1}+1\right)=1 / 7=14.3 \%$.

${ }^{\mathrm{b}}$ Equals $0.5 \times$ probability of III: 4 .

${ }^{\mathrm{c}}$ Equals $0.5 \times$ probability of II: 1 .

${ }^{\mathrm{d}}$ Equals $0.25 \times$ probability of II: 1 . 
Table 2 Bayesian calculation of probability that individual II: 1 is a carrier of the HD gene, given that $n$ children of individual III: 4 in Figure 1 are established noncarriers (and no other test results available), taking age-dependent penetrance for individual II: 1 into account; $p$ is the estimated age-adjusted probability that individual II: 1 is a carrier of the HD gene

\begin{tabular}{|c|c|c|c|}
\hline \multirow{3}{*}{ Age-adjusted probability } & \multicolumn{2}{|r|}{ II:1 carrier } & \multirow{3}{*}{$\begin{array}{c}\text { II:1 noncarrier } \\
1-\mathrm{p} \\
\text { III:4 noncarrier }\end{array}$} \\
\hline & \multicolumn{2}{|r|}{$\mathrm{p}$} & \\
\hline & III:4 carrier & III:4 noncarrier & \\
\hline Probability that event occurs & $1 / 2$ & $1 / 2$ & 1 \\
\hline $\begin{array}{l}\text { Probability that } n \text { children of III: } 4 \\
\text { are noncarriers }\end{array}$ & $1 / 2^{n}$ & 1 & 1 \\
\hline Joint odds & $p /\left(2^{n+1}\right)$ & $p / 2$ & $1-p$ \\
\hline Posterior probability & {$\left[p /\left(2^{n+1}\right)\right] /\left[p /\left(2^{n+1}\right)+p / 2+(1-p)\right]$} & $(p / 2) /\left[p /\left(2^{n+1}\right)+p / 2+(1-p)\right]$ & \\
\hline $\begin{array}{l}\text { Probability that individual II: } 1 \text { is a } \\
\text { have been tested mutation negativ }\end{array}$ & arrier, given that $n$ children of III: 4 & $\begin{array}{c}{\left[p /\left(2^{n+1}\right)\right] /\left[p /\left(2^{n+1}\right)+p / 2+(1-p)\right]+(p / 2) /[p /} \\
\left.\left(2^{n+1}\right)+p / 2+(1-p)\right]=p *\left(1+2^{n}\right) /\left[p *\left(1-2^{n}\right)+2^{n+1}\right]^{\#}\end{array}$ & \\
\hline
\end{tabular}

\#In case of $p=1 / 2$ (prior probability), this can be rewritten as $1 / 2 *\left(1+2^{n}\right) /\left[1 / 2 *\left(1-2^{n}\right)+2^{n+1}\right]=\left(1+2^{n}\right) /\left(1-2^{n}+2^{n+2}\right)=\left(2^{n}+1\right) /\left(3 * 2^{n}+1\right)($ see Table 1).

In conclusion, accurate assessment of conditional probabilities in clinical genetics is important for individuals at risk for hereditary disorders with Mendelian transmission patterns, not the least because other relatives who might also benefit from genetic counselling can thus be identified. The formulae can be used as an aid to calculating conditional genetic risks in relevant situations provided that the simplified assumptions made in this paper are met. Furthermore, care must be taken that those using these formulae understand the underlying principles well in order to prevent misuse.

\section{References}

Bonke B, Tibben A, Lindhout D, Clarke AJ, Stijnen T (2005). Genetic risk estimation by healthcare professionals. Med $J$ Austr 182: 116-118.
Bonke B, Tibben A, Lindhout D, Stijnen T (2002). Favourable mutation test outcomes for individuals at risk for Huntington disease change the perspectives of first-degree relatives. Hum Genet 111: 297-298.

Bridge PJ (1994). The Calculation of Genetic Risks. Johns Hopkins University Press: Baltimore.

Claus EB, Risch N, Thompson WD (1993). The calculation of breast cancer risk for women with a first degree family history of ovarian cancer. Breast Cancer Res Treat 28: 115-120.

Gelehrter TD, Collins FS, Ginsburg D (1998). Principles of Medical Genetics, 2nd edn. Williams \& Wilkins: Baltimore.

Harper PS, Newcombe RG (1992). Age at onset and life table risks in genetic counselling for Huntington's disease. J Med Genet 29: 239-242.

Maat-Kievit A, Losekoot M, Zwinderman K, Vegter-van der Vlis M, Belfroid R, Lopez F et al (2002). Predictability of age at onset in Huntington disease in the Dutch population. Medicine (Baltimore) 81: 251-259. 